\title{
Microfluidic models of neuromuscular junctions
}

\section{Editorial}

The neuromuscular junction (NMJ) acts as a bridge for the signals to travel between a motor neuron and a muscle fiber. The setup as a whole is referred to a motor unit which is virtually responsible for all the motor functions that includes breathing, talking, respiratory activities etc. The need for an in vitro platform to monitor the neuromuscular junction arises because of the loss of NMJs in neurodegenerative diseases. The escalating deterioration and loss of function of neurons lead to some destructing conditions which are collectively known as neurodegenerative diseases. ${ }^{1}$ Neuromuscular disorders can be segregated into motor-neuron diseases like amyotrophic lateral sclerosis (ALS), ${ }^{2}$ spinal muscular atrophy (SMA) $;{ }^{3}$ muscular ailments like Duchenne's and Becker's muscular dystrophies ${ }^{4}$ auto-immune diseases like myasthenia gravis, ${ }^{5}$ to name a few. To comprehend and unveil the basic functioning of a neuromuscular junction and the disorders associated with it requires modeling of NMJ on an in vitro platform that is able to delineate fundamental aspects related to formation and maintenance of NMJ.

Traditional culture platforms like petri dishes and well plates do not offer the liberty to fine-tune the events at micro scale level. Coincidently, this freedom is bestowed upon us by the emerging technology termed as microfluidics. The science and technology of microfluidics helps in controlling the amount of reagent used, fluid flow, co-culture by compartmentalization and cell-cell interaction in a spatial and temporal way. ${ }^{6}$ Amongst the most conventional compartmentalized platforms for recreation of in vitro neuromuscular junctions are Campenot chamber ${ }^{7}$ and Ivins' chamber. ${ }^{8}$ Microfluidic platforms holds great potential in biological research considering its miniaturization and automation abilities. Vilmont et al. ${ }^{9}$ have described a method for the development of heterologous co-culture system. ${ }^{9}$ 3D growth of neuron-muscle structures was facilitated by the use of Matrigel. This system although did not use any microfluidic platform, but proposed a method which can be easily integrated with microfluidic devices. Recently, Uzel et al. ${ }^{10}$ have designed a compartmentalized microfluidic platform for co-culture of myoblasts and motor neurons. The proposed microenvironment of the device enables physical separation of the two compartments. The device also exploits the use of optically excitable motor neurons, differentiated from mouse embryonic stem cells, for transmitting information to muscle strips being anchored by flexible pillars. ${ }^{10}$ To reveal the mechanisms behind devastating neurodegenerative disease, the interaction between neuron-neuron, neuron-astrocyte and neuronmuscle should be identified distinctly. To showcase the influence of these cell types on each other Gao et al. ${ }^{11}$ has put forward an experimental setup by integrating microfluidics with micropatterning and genetically encoded calcium indicators. ${ }^{11}$ Another successful attempt of microfluidic technology for neurobiology research has been conducted by Ionescu et al. ${ }^{12}$ here, they have made good use of GFP labelled motor neurons to innervate muscle cells derived from satellite progenitor cells. Formation of grooves for the passage of axons inhibits the interruption of any unwanted experimental particle in the neuron-muscle route. ${ }^{12}$ Blizzard et al. ${ }^{13}$ have demonstrated the use of compartmentalized microfluidic device for site-specific excitotoxin exposure. It is believed that excitotoxic mechanisms might play an important role in motor neuron death in ALS. The
Volume 2 Issue 3 - 2017

\author{
Suruchi Poddar, Sanjeev Kumar Mahto \\ Indian Institute of Technology, India
}

Correspondence: Sanjeev Kumar Mahto,Tissue Engineering and Biomicrofluidics Laboratory, School of Biomedical Engineering, Indian Institute of Technology (Banaras Hindu University),Varanasi 221005, Uttar Pradesh, India, Email skmahto.bme@iitbhu.ac.in

Received: March 04, 2017 | Published: April 10, 2017

findings of this experiment recommend that excitotoxicity in the spinal cord of individuals with ALS might result in a transneuronal degeneration, leading to permanent damage of the neurons, followed by its degeneration. ${ }^{13}$ To study the synaptic communication among neuronal populations, Renault et al. ${ }^{14}$ have devised an in vitro platform combining microfluidics, optogenetics and calcium imaging. The micro-compartments in the device are coupled with optical fibers for independent control of Channelrhodopsin-2 protein that is being used as a calcium imaging tool. The device geometry helps in preventing the cross talk between neuronal populations.${ }^{14} \mathrm{Li}$ et al. ${ }^{15}$ have exploited the use of microfluidic device for amperometric measurements of neurotransmitter release. The microscale environment of the device helps in the formation of in vitro neural networks between superior cervical ganglion and effector smooth muscle cells. This device claims to be the first in monitoring in situ chemical synaptic transmission under physiologically relevant conditions. ${ }^{15}$ Tong et al. ${ }^{16}$ \& Zahavi et al. ${ }^{17}$ have achieved the bidirectional communication between motor neurons and muscle cells for the formation and healthy maintenance of neuromuscular junction. ${ }^{16,17}$ The devices mentioned above hold great potential in high throughput drug screening, therapy design, conducting physiological and mechanistic studies, replacing animal models, soft-robotic actuators ${ }^{18}$ etc.. ${ }^{19-26}$

\section{Acknowledgements}

None.

\section{Conflict of interest}

The author declares no conflict of interest.

\section{References}

1. Vann KT, Xiong ZG. Optogenetics for neurodegenerative diseases. Int $J$ Physiol Pathophysiol Pharmacol. 2016;8(1):1-8.

2. Lee S, Huang EJ. Modeling ALS and FTD with iPSC-derived neurons. Brain Res. 2017;1656:88-97.

3. Edens BM, Ajroud Driss S, Ma L, et al. Molecular mechanisms and animal models of spinal muscular atrophy. Biochim Biophys Acta. $2015 ; 1852(4): 685-692$.

4. Flanigan KM. Duchenne and Becker muscular dystrophies. Neurol Clin 2014;32(3):671-688.

5. Ha JC, Richman DP. Myasthenia gravis and related disorders: Pathology and molecular pathogenesis. Biochim Biophys Acta. 2015;1852(4):651657. 
6. Li R, Lv X, Zhang X, et al. Microfluidics for cell-cell interactions: A review. Frontiers of Chemical Science and Engineering. 2016;10(1):90 98.

7. Campenot RB. Development of sympathetic neurons in compartmentalized cultures. II. Local control of neurite survival by nerve growth factor. Dev Biol. 1982;93(1):13-21.

8. Ivins KJ, Bui ET, Cotman CW. Beta-amyloid induces local neurite degeneration in cultured hippocampal neurons: evidence for neuritic apoptosis. Neurobiol Dis. 1998;5(5):365-378.

9. Vilmont V, Cadot B, Ouanounou G, et al. A system to study mechanisms of neuromuscular junction development and maintenance. Development dev. 2016;143(13):2464-2477.

10. Uzel SG, Platt RJ, Subramanian V, et al. Microfluidic device for the formation of optically excitable, three-dimensional, compartmentalized motor units. Sci Adv. 2016;2(8):e1501429.

11. Gao Y, Broussard J, Haque A, et al. Functional imaging of neuronastrocyte interactions in a compartmentalized microfluidic device. Microsyst Nanoeng. 2016. 2 p.

12. Ionescu A, Zahavi EE, Gradus T, et al. Compartmental microfluidic system for studying muscle-neuron communication and neuromuscular junction maintenance. Eur J Cell Biol. 2016;95(2):69-88.

13. Blizzard CA, Southam KA, Dawkins E, et al. Identifying the primary site of pathogenesis in amyotrophic lateral sclerosis-vulnerability of lower motor neurons to proximal excitotoxicity. Dis Model Mech. 2015;8(3):215-224.

14. Renault R, Sukenik N, Descroix S, et al. Combining microfluidics, optogenetics and calcium imaging to study neuronal communication In Vitro. PLoS ONE. 2015;10(4):e0120680.

15. Li YT, Zhang SH, Wang XY, et al. Real-time monitoring of discrete synaptic release events and excitatory potentials within self-reconstructed neuromuscular junctions. Angew Chem Int Ed Engl. 2015;54(32):93139318
16. Tong Z, Seira O, Casas C, et al. Engineering a functional neuro-muscular junction model in a chip. RSC Adv. 2014;97:54788-54797.

17. Zahavi EE, Ionescu A, Gluska S, et al. A compartmentalized microfluidic neuromuscular co-culture system reveals spatial aspects of GDNF functions. J Cell Sci. 2015;128(6):1241-1252.

18. Morimoto Y, Kato-Negishi M, Onoe H, et al. Three-dimensional neuron-muscle constructs with neuromuscular junctions. Biomaterials 2013;34(37):9413-9419.

19. Cheng J, Chang T, Bhattacharjee N, et al. Neuromuscular synaptogenesis in an open chamber microfluidic device. WA, United States. 2011;1:356358.

20. Colosimo A, Frank C. What can we learn by connecting neurons in a nanodevice? Biophys Bioeng Lett. 2010;3(2):1-10.

21. Guo X, Das M, Rumsey J, et al. Neuromuscular junction formation between human stem-cell-derived motoneurons and rat skeletal muscle in a defined system. Tissue Eng Part C Methods. 2010;16(6):1347-1355.

22. Park HS, Liu S, Mc Donald J, et al. Neuromuscular junction in a microfluidic device. Conf Proc IEEE Eng Med Biol Soc. 2013;2013:28332835.

23. Smith AS, Long CJ, Pirozzi K,et al. A functional system for high-content screening of neuromuscular junctions in vitro. Technology (Singap World Sci). 2013;1(1):37-48.

24. Southam KA, King AE, Blizzard CA, et al. Microfluidic primary culture model of the lower motor neuron-neuromuscular junction circuit. $J$ Neurosci Methods. 2013;218(2):164-169.

25. Stirman JN, Brauner M, Gottschalk A, et al. High-throughput study of synaptic transmission at the neuromuscular junction enabled by optogenetics and microfluidics. J Neurosci Methods. 2010;191(1):90-93.

26. Wang J, Ren L, Li L, et al. Microfluidics: a new cosset for neurobiology. Lab Chip. 2009;9(5):644-652. 